\title{
Comparación de métodos para el análisis de coliformes totales y fecales en muestras de agua mediante la técnica de Número Más Probable (NMP)
}

\author{
Mauricio Redondo-Solano y María Laura Arias Echandi \\ Laboratorio de Microbiología de Alimentos y Aguas, Facultad de Microbiología, Universidad de Costa Rica, San José; maria.ariasechandi@ucr.ac.cr
}

Recibido 6-V-2011 Corregido 7-VI-2011 Aceptado 21-VI-2011

\begin{abstract}
Comparisson of methods for the analysis of total and fecal coliforms in water samples using the Most Probable Number technique. The basic microbiological analysis of water includes the determination of total and fecal coliforms. The presence or abscense of fecal coliforms in water defines its potability. The main objective of this work was to design and execute a comparison scheme of two techniques for the determination of coliforms in water, applying modifications to the reference technique of the Most Probable Number, derived from the Standard Methods for the Examination of Water and Wastewater. The modifications included the type of media used for the presumptive phase and the use of an air incubator instead of a water bath for the confirmatory phase. At the same time, the effect of using calibrated automatic pipettes and calibrated glass pipettes was also compared. Artificial water samples, elaborated with standard laboratory material and inoculated with Escherichia coli ATCC 25922 and environmental samples, were used. The alternative methodology shows a superior performance only when inoculation is low. There was no difference between the two volumetric systems.
\end{abstract}

KEY WORDS

Total coliforms, fecal coliforms, water, Most Probable Number.

\section{RESUMEN}

El análisis microbiológico básico del agua comprende la determinación de coliformes totales y fecales. La ausencia o presencia de los coliformes fecales en el agua define su potabilidad. El objetivo del presente estudio consistió en el diseño y ejecución de un esquema de comparación de dos técnicas para la determinación de coliformes en aguas, a partir de modificaciones aplicadas sobre la técnica de referencia de Número Más Probable (NMP) derivada del Standard Methods for the Examination of Water and Wastewater. Específicamente, las modificaciones incluyeron el tipo de medio utilizado en la fase presuntiva de la técnica y el uso de incubadora de aire, alternativo al baño de maría en la fase confirmatoria. Simultáneamente, se comparó el efecto del uso de equipo volumétrico tipo micropipeta automática calibrada o pipetas de vidrio estériles calibradas. Para tal efecto, se utilizaron muestras de agua artificiales, elaboradas con material del laboratorio e inoculadas con una cepa de Escherichia coli ATCC 25922 así como muestras ambientales. La metodología alternativa tiene un rendimiento superior solamente cuando el nivel de inóculo en las muestras es bajo. No se determinó ninguna diferencia estadísticamente significativa entre los dos sistemas de medición volumétrica.

PALABRAS CLAVE

coliformes totales, coliformes fecales, agua, Número Más Probable.
El análisis microbiológico básico del agua comprende la determinación de coliformes totales y fecales. Esta determinación es importante porque brinda información valiosa en el estudio de cambios que se puedan presentar en las poblaciones microbianas presentes en las fuentes de agua (Abbaszadegan 2006). De la misma manera, la ausencia o presencia de los coliformes fecales en el agua define su potabilidad, al ser organismos indicadores de contaminación fecal.

La importancia del monitoreo microbiológico del agua ha motivado el desarrollo de un número importante de técnicas de análisis basadas en principios variados. Uno de los métodos comúnmente empleados en varios países es el basado en la técnica de los tubos múltiples de fermentación o número más probable (NMP; código ISO 9308-2:1990). No obstante, se pueden emplear nuevas y distintas metodologías de ensayo, siempre y cuando éstas demuestren un comportamiento igual o superior al método definido como de referencia.

Se define validación como la demostración de que los resultados obtenidos por un método alternativo son comparables a aquellos obtenidos por el método de 
referencia, utilizando los protocolos estadísticos apropiados (Feldsine et al. 2002).

Durante el proceso de validación es posible identificar si la metodología nueva o modificada se acepta para su uso, en tanto demuestre equivalencia con el método de referencia, entendiéndose ésta como una diferencia no significativa en los resultados emitidos por ambos métodos, o se determine que el nuevo ensayo demuestra un comportamiento superior. Si en el proceso de validación el nuevo ensayo demuestra un recuento significativamente más bajo que el de referencia, se debe rechazar (Barrell et al. 2002). La base del método de referencia es fundamental, pues es esta metodología la que posee reconocimiento internacional sobre su desempeño, al analizar $r$ la muestra o analito de interés, en el caso de este trabajo, la presencia de coliformes totales y fecales en agua.

En el presente trabajo se estudió el diseño y ejecución de un esquema de comparación de dos técnicas para la determinación de coliformes en aguas, a partir de modificaciones aplicadas sobre la técnica de referencia de Número Más Probable (NMP) derivada del Standard Methods for the Examination of Water and Wastewater, edición 21, publicada por la American Public Health Association en el año 2005. Específicamente, las modificaciones incluyen el tipo de medio utilizado en la fase presuntiva de la técnica y el uso de incubadora de aire alternativo al baño de maría en la fase confirmatoria. Simultáneamente, se comparó el efecto del uso de equipo volumétrico tipo micropipeta automática calibrada con puntas estériles o pipetas de vidrio estériles calibradas.

\section{METODOLOGÍA}

\section{Preparación del cultivo microbiológico}

Se utilizó una cepa de referencia de E. coli (ATCC 25922) mantenida en congelación $\left(-80^{\circ} \mathrm{C}\right)$ empleando Caldo Infusión Cerebro y Corazón $\left(\mathrm{Oxoid}^{\circledR}\right)$ suplementado con glicerol (50\%) y reconstituida en Caldo Tripticasa Soya (CTS) $\left(\mathrm{Oxoid}^{\oplus}\right)$, por 18 horas, a $35^{\circ} \mathrm{C}$. La población bacteriana final fue de alrededor $9 \log$ UFC/mL.

\section{Obtención y preparación de las muestras}

Se trabajó con muestras artificiales elaboradas con material estándar de laboratorio y la cepa de E. coli ATCC 25922. Siguiendo los fundamentos de algunos esquemas de validación en microbiología (Weitzel 2007) se prepararon, con esta cepa de referencia, muestras inoculadas artificialmente, empleando agua destilada estéril. Se obtuvieron tres muestras de este tipo, cada una consistente de un volumen final de $2 \mathrm{~L}$, que abarcaron tres niveles de inóculo diferentes: alto (con concentraciones mayores a $250 \mathrm{NMP} / 100 \mathrm{~mL}$ ), medio (concentraciones entre 10 y $250 \mathrm{NMP} / 100 \mathrm{~mL}$ ) y bajo (concentraciones entre 2 y $9 \mathrm{NMP} / 100 \mathrm{~mL}$ ), procurando que existieran diferencias de por lo menos un logaritmo entre el inóculo bajo y los demás, basándose en los valores brindados por la tabla de NMP. El inóculo bajo alcanza el límite para la definición de la potabilidad del agua (2NMP de coliformes fecales $/ 100 \mathrm{~mL}$ ). Del cultivo madre original se tomaron alícuotas para realizar diluciones en tubos con agua peptonada estéril al $0,1 \%$, en cantidades suficientes de forma tal que, a partir de la dilución más alta realizada, se tomara un mililitro para sembrar en la matriz de $2 \mathrm{~L}$, alcanzando la concentración de interés. Simultáneamente, a partir de las diluciones en agua peptonada y el cultivo original se inocularon placas de Agar Bilis Rojo Violeta para confirmar el tamaño de la población microbiana; estas placas se incubaron a $44,5^{\circ} \mathrm{C}$ durante 24 horas. A las muestras inoculadas se les agregó cloro comercial, de forma tal que se alcanzara una concentración aproximada de $3 \mathrm{mg} / \mathrm{L}$, simulando la cantidad presente en el agua del sistema público de distribución. Después de una exposición máxima de 15 minutos, el cloro presente se neutralizaba con pastillas de tiosulfato de sodio $\left(\mathrm{Oxoid}^{\oplus}\right)$; el objetivo de esta preparación es el de imitar las condiciones derivadas de un mal tratamiento (Public Health Laboratory Service 1980, Barrell et al. 2002). En todo momento las muestras fueron mantenidas en agitación constante por medio de un agitador mecánico con el fin de asegurar la homogeneidad de la muestra. Diferentes volúmenes de agua destilada estéril fueron empleados como muestras negativas en las distintas fases del trabajo.

Para la obtención de las muestras de agua utilizadas en la segunda fase de comparación, se aplicaron las especificaciones de muestreo detalladas en el Standard Methods for the Examination of Water and Wastewater (procedimiento 9060 A) (APHA 2005). Se incluyen muestras ambientales, derivadas de fuentes urbanas y del sistema público de distribución, recolectadas en distintos puntos de la provincia de San José. Se combinó la presencia de muestras desinfectadas o sin desinfectar. El volumen mínimo de agua recolectado fue de $200 \mathrm{~mL}$ de forma que se pudiera realizar el montaje de la misma muestra comparando las diferentes variantes metodológicas. Se emplearon un total de 50 muestras.

\section{Análisis microbiológico}

En la determinación de la carga microbiana en las muestras seleccionadas se emplearon las metodologías del Standard Methods for the Examination of Water and 
Wastewater (APHA 2005) para el análisis de coliformes totales y fecales por el método de tubos múltiples de fermentación (procedimientos 9-49 y 9-56). Estas metodologías son las que se toman en el presente estudio como normalizadas o de referencia.

Las modificaciones incluidas en el método alternativo consisten en el uso de Caldo Lactosado (Oxoid ${ }^{\circledR}$ ) doble y simple durante la fase presuntiva, en lugar de Caldo Lauril Triptona; además del uso de una incubadora de aire (Memmert) en la incubación del Caldo EC (Oxoid $\left.{ }^{\oplus}\right)$ para la fase confirmatoria de coliformes fecales ( 24 horas a $44,5^{\circ} \mathrm{C}$ ).

\section{Protocolo de comparación}

El esquema de comparación empleado se formuló tomando como base las indicaciones del Standard Methods for the examination of water and wastewater (APHA 2005) y la norma ISO/TR 13843 "Guía para la validación de métodos microbiológicos en aguas. El protocolo se dividió en varias partes: (1) Uso de Caldo Lactosado en fase presuntiva para coliformes totales y fecales: para esta parte se trabajaron 10 muestras de concentración alta de $E$. coli (cepa de referencia ATCC 25922), 10 con concentración media, 10 con concentración baja y 10 negativas. Cada una de las concentraciones se montó 10 veces con el procedimiento de referencia y el alternativo. En la técnica estandarizada se utilizó Caldo Lauril Triptona para la fase de enriquecimiento; en la técnica a validar se utilizó Caldo Lactosado. (2) Uso de incubadora de aire en fase confirmatoria de coliformes fecales: se trabajó con 10 muestras de concentración alta de E. coli (cepa de referencia ATCC 25922), 10 con concentración media, 10 con concentración baja y 10 negativas. Cada una de las concentraciones se montó 10 veces con el procedimiento de referencia y el alternativo. En la técnica estandarizada se utiliza un Baño María (Memmert) a $44,5+0,2^{\circ} \mathrm{C}$ durante 24 horas. En la técnica a validar se empleó una incubadora de aire a $44,5+$ $0,2^{\circ} \mathrm{C}$ durante 24 horas, para confirmar los coliformes fecales. (3) Comparación incluyendo las dos variables juntas: en este punto se analizaron 50 muestras de origen natural; cada una de ellas se montó con la técnica de referencia y la alternativa. En el montaje de la técnica alternativa se incluyó el uso de Caldo Lactosado en la fase presuntiva para ambas determinaciones y el empleo de una incubadora de aire a $44,5+0,2^{\circ} \mathrm{C}$ en la fase confirmatoria para coliformes fecales. De las muestras que resultaron positivas se eligieron 15 para realizarles la confirmación de los resultados obtenidos. En la metodología de coliformes totales la confirmación se realizó a partir de cada tubo positivo de Caldo Bilis Verde Brillante $\left(\mathrm{Oxoid}^{\circledR}\right)$, rayando placas con agar MacConkey (Oxoid ${ }^{\circledR}$ ), realizando una tinción de Gram e inoculando un tubo secundario de Caldo Lauril Triptona
Simple (Oxoid $\left.{ }^{\circledast}\right)$. La confirmación se consideraba oportuna cuando se observaban colonias rojas en el agar, bacilos Gram negativos en la tinción y la generación de gas y turbidez en los tubos secundarios de Caldo Lauril Triptona. En la confirmación de coliformes fecales se tomaron los tubos de Caldo EC con reacción positiva y se rayaron placas con Agar Bilis Rojo Violeta (Oxoid ${ }^{\oplus}$ ), placas de Agar Sangre y se realizó tinción de Gram. A partir de las colonias crecidas en Agar Sangre en 24 horas se inocularon tubos con Caldo Triptona para efectuar la prueba de indol. La confirmación positiva para E. coli se determinó al observar bacilos Gram negativos, colonias rojas con precipitado opaco en el Agar Bilis Rojo Violeta (después de una incubación de 24 horas a $44,5+0,2^{\circ} \mathrm{C}$ ) y una prueba de indol positiva. (4) Comparación del método al usar dos sistemas volumétricos diferentes en la inoculación de las muestras: Se trabajó con 5 muestras de concentración alta de E. coli (cepa de referencia ATCC 25922), 5 con concentración media, 5 con concentración baja y 5 negativas. Cada muestra se inoculó de forma simultánea con la técnica alternativa empleando una micropipeta automática calibrada con puntas estériles y pipetas de vidrio estériles calibradas.

\section{Análisis estadístico de los datos}

El análisis numérico de los datos obtenidos en la validación se basa en las instrucciones referidas en la guía ISO 17994. Para la comparación de métodos de Número Más Probable, se determina la diferencia relativa entre cada una de las mediciones pareadas. Se calcula además la incertidumbre expandida (U) y con ello los límites de confianza superior e inferior al 95\%. Los criterios de análisis establecen que dos métodos son equivalentes si los recuentos no difieren significativamente de cero y tanto el límite superior como el inferior del intervalo de confianza al $95 \%$ de la media no superan un nivel máximo de desviación aceptable (valor $\mathrm{D}$, que en este caso es de 10\%) En el análisis de muestras de referencia y el empleo de sistemas volumétricos diferentes se determinó la precisión del método, mediante el cálculo del valor de referencia R (International Organization for Standardisation 1996, International Organization for Standardisation 2005). Por medio del uso de una t de Student se determinó si las diferencias entre los recuentos en las muestras de referencia en los distintos intervalos de estudio muestran diferencias significativas (en los casos donde $p<0,05$ ). Para efectos de cálculo fueron omitidos todos aquellos resultados donde ambas muestras fueron negativas. Si el resultado de análisis fue de $>1600 \mathrm{NMP} / 100 \mathrm{~mL}$ para una muestra se asignó un valor arbitrario de $1650 \mathrm{NMP} / 100 \mathrm{~mL}$ para efectuar los cálculos correspondientes. 


\section{RESULTADOS}

\section{Comparación de caldos en la fase presuntiva y del tipo de incubación realizada}

Con el fin de evaluar el efecto de la variación en el caldo de enriquecimiento y del tipo de incubación a $44,5^{\circ} \mathrm{C}$, se realizó la comparación estadística correspondiente para el logaritmo natural del NMP obtenido para las muestras inoculadas artificialmente y las naturales. En el Cuadro 1, se detallan las diferencias en los promedios obtenidos a partir del método tradicional y el alternativo al analizar muestras artificiales:

Los resultados obtenidos demuestran que las variaciones aplicadas sobre la metodología de ensayo ejercen un efecto estadísticamente significativo en los resultados de comparación $(p<0,05)$, observándose un mayor rendimiento obtenido a partir del método de referencia que el alternativo. La metodología alternativa muestra un rendimiento superior solamente cuando el nivel de inóculo en las muestras es bajo.

Analizando el porcentaje de positividad de los tubos empleados se demuestra que el desempeño de la prueba confirmatoria para coliformes fecales se incrementa cuando se emplea caldo lactosado como medio de enriquecimiento, tanto a niveles bajos y medios. Asimismo, el porcentaje de positividad fue superior para la metodología alternativa cuando una incubadora convencional fue empleada en la fase confirmatoria, en comparación con el uso del baño maría.

\section{CUADRO 1}

Comparación del promedio de los valores de InNMP/100mL obtenidos para coliformes totales y fecales en muestras artificiales según las variaciones al método introducidas

\begin{tabular}{lccc}
$\begin{array}{c}\text { Nivel } \\
\text { inóculo }\end{array}$ & $\begin{array}{c}\text { Coliformes } \\
\text { Totales Pres }^{\mathrm{a}}\end{array}$ & $\begin{array}{c}\text { Coliformes } \\
\text { Fecales Pres }\end{array}$ & $\begin{array}{c}\text { Fecales } \\
\text { Conf }^{\mathrm{b}}\end{array}$ \\
\hline Alto & $1,26^{\mathrm{c}} \pm 1,53$ & $1,43 \pm 1,64$ & $-0,77^{\mathrm{d}} \pm 1,02$ \\
Medio & $0,69 \pm 0,69$ & $-3,31 \pm 2,07$ & $-3,13 \pm 2,22$ \\
Bajo & $-0,31 \pm 0,31$ & $-0,51 \pm 0,22$ & $-0,35 \pm 0,21$
\end{tabular}

aResultado de la comparación utilizando Caldo Lactosado en el método alternativo durante la fase presuntiva.

besultado de la comparación utilizando incubadora de aire en el método alternativo durante la fase confirmatoria.

'Resultado positivo indica un desempeño superior en el método de referencia.

${ }^{d}$ Resultado negativo indica un desempeño superior en el método alternativo.
En el Cuadro 2, se muestra el comportamiento de las muestras naturales analizadas, es evidente el comportamiento superior al utilizar la metodología de referencia.

De los datos anteriores se puede observar como el rendimiento del método de referencia se incrementa notablemente durante el análisis de muestras naturales, en comparación con lo observado en las muestras artificiales del Cuadro 1. El comportamiento del análisis de los coliformes totales en las muestras naturales siguió el mismo patrón de las muestras artificiales, es decir, un comportamiento superior del método alternativo solamente en el nivel de población bacteriano bajo. Sin embargo, para los coliformes fecales, el método alternativo solamente se comportó superior a este mismo nivel de inóculo bajo, situación diferente a lo observado con el primer grupo de muestras. El análisis estadístico demuestra que todas las diferencias encontradas en estos resultados son estadísticamente significativas $(p<0,05)$.

El cálculo de la incertidumbre expandida y de los límites de confianza superior e inferior muestran que el proceso de comparación no es concluyente para el conjunto de muestras naturales (valores no mostrados) ya que los valores de ambos límites de confianza fueron inferiores al valor calculado $\mathrm{U}$.

\section{Efecto del uso de equipo volumétrico tipo micropipeta automática calibrada o pipeta de vidrio estéril calibrada}

No se determinó ninguna diferencia estadísticamente significativa entre los dos sistemas de medición volumétrica empleados utilizando Caldo Lactosado en la fase presuntiva del ensayo. El método es lo suficientemente robusto en cuanto a precisión volumétrica con ambos sistemas, pues para ambas determinaciones (coliformes totales y fecales) el valor promedio de las diferencias fue menor al valor de criterio R estimado (3,70 para coliformes fecales y 3,99 para coliformes totales).

\section{DISCUSIÓN}

En microbiología de aguas existen muchos estudios tendientes a la comparación de nuevos métodos para el análisis de coliformes dada la importancia que esta determinación reviste en varios campos. La mayoría de estas comparaciones toma como base los métodos estándar definidos por la OMS, fundamentados en diferentes principios como la filtración en membrana (McDaniels et al. 1987), transformación enzimática de sustratos (Clark et al. 1991), cultivo en placas (Garcia-Armisen et al. 2007) y el Número Más Probable (Eckner 1998). Estas comparaciones 


\section{CUADRO 2}

Datos de comparación para muestras naturales empleando ambas variables metodológicas simultáneamente

\begin{tabular}{|c|c|c|c|c|c|}
\hline $\begin{array}{c}\text { Tipo } \\
\text { de muestra }\end{array}$ & $\begin{array}{l}\text { Número } \\
\text { Total }\end{array}$ & $\begin{array}{l}\text { Promedio Referencia } \\
\text { (InNMP/100mL) }\end{array}$ & $\begin{array}{c}\text { Promedio } \\
\text { Alternativo } \\
\text { (InNMP/100mL) }\end{array}$ & $\begin{array}{c}\text { Diferencia } \\
\text { Promedio } \\
\text { (InNMP/100mL) }\end{array}$ & $\begin{array}{l}\text { Método con } \\
\text { el mayor } \\
\text { rendimiento }\end{array}$ \\
\hline $\begin{array}{l}\text { Totales } \\
\text { (todas las muestras) }\end{array}$ & 41 & $5,91 \pm 2,81$ & $5,48 \pm 1,54$ & $0,43 \pm 2,16$ & Referencia \\
\hline $\begin{array}{l}\text { Fecales } \\
\text { (todas las muestras) }\end{array}$ & 39 & $6,53 \pm 1,76$ & $5,32 \pm 1,60$ & $1,21 \pm 0,15$ & Referencia \\
\hline Totales Carga Alta & 28 & $7,23 \pm 0,39$ & $6,50 \pm 1,70$ & $0,73 \pm 1,68$ & Referencia \\
\hline Totales Carga Media & 9 & $3,71 \pm 1,16$ & $3,64 \pm 2,78$ & $0,07 \pm 2,25$ & Referencia \\
\hline Totales Carga Baja & 4 & $1,18 \pm 1,00$ & $3,19 \pm 2,93$ & $-2,01 \pm 3,61$ & Alternativo \\
\hline Fecales Carga Alta & 26 & $7,24 \pm 0,40$ & $6,58 \pm 0,97$ & $0,66 \pm 1,02$ & Referencia \\
\hline Fecales Carga Media & 6 & $3,86 \pm 1,01$ & $3,60 \pm 3,07$ & $1,26 \pm 2,46$ & Referencia \\
\hline Fecales Carga Baja & 7 & $1,35 \pm 0,95$ & $1,78 \pm 2,13$ & $-0,43 \pm 2,07$ & Alternativo \\
\hline
\end{tabular}

han facilitado el desarrollo de metodologías rápidas, de fácil manejo, capaces de emitir resultados confiables y superiores en ciertos casos a los métodos de referencia. Sin embargo, la aplicación de estas técnicas supone costos elevados para los laboratorios que optan por su utilización (Moldenhauer 2005) razón por la que en ciertos casos se prefiere el mejoramiento de las técnicas estándar establecidas con anterioridad. Uno de los mayores problemas en el uso de las técnicas estándar consiste en que los laboratorios los emplean basados en el reconocimiento internacional del que han gozado a través de los años, sin importar que se haya hecho poco para demostrar su desempeño (Sartory 2005)

En el Cuadro 1, se muestran los resultados obtenidos en el análisis de muestras inoculadas artificialmente. Los resultados obtenidos ponen de manifiesto que el método alternativo muestra un desempeño aceptable pues es superior al método de referencia en varias de las condiciones señaladas. En varias de las muestras analizadas, el medio Lauril Triptona tuvo una menor eficiencia en la recuperación de $E$. coli, situación importante al tomar en cuenta que muchos resultados denotan ausencia de coliformes fecales, definiendo la fuente de agua falsamente como potable (Abbaszadegan 2006). Al ser este medio de cultivo más restrictivo para el crecimiento que el Caldo Lactosado podría ejercer un efecto negativo en el crecimiento de $E$. coli sometida al estrés del agente desinfectante; el efecto mayor observado en relación con la determinación de coliformes totales puede entenderse en el uso de un medio de cultivo (Caldo EC) y temperaturas de incubación $\left(44,5^{\circ} \mathrm{C}\right)$ más restrictivas. Pitkanen et al. (2007) encontraron, de la misma forma, rendimientos superiores en métodos alternativos cuando se analizan coliformes fecales. La observación de que el NMP de coliformes fecales presenta diferencias significativas cuando se emplean métodos de incubación diferentes, revela que el efecto del medio de cultivo en fase presuntiva no es el único factor determinante en las diferencias observadas. Una de las desventajas en el uso de las incubadoras de aire es la falta de homogeneidad en varios de sus compartimentos durante el periodo de incubación (Barrell et al. 2002). Estas fluctuaciones no siempre pueden ser monitoreadas y en muchos casos podrían dirigirse hacia un incremento de la temperatura. Estas fluctuaciones pueden haber favorecido el crecimiento de $E$. coli en la incubadora de aire; la literatura reporta que incubar el caldo EC a mayores temperaturas (por ejemplo a $45,5^{\circ} \mathrm{C}$ ) podría aumentar la especificidad hacia los coliformes fecales (Pouch 2001, Beauchamp et al. 2006). En todo caso, las diferencias observadas pueden radicar en un efecto de dispersión de la población bacteriana a la hora de montar las muestras; diversos estudios han demostrado que dentro de las principales características de las muestras de agua se encuentra la distribución al azar de las bacterias, aumentando la imprecisión de las mediciones y la diferencia en los resultados (Barrell et al. 2002). Otro factor mencionado es la variabilidad misma de las técnicas de NMP, consideradas por algunos autores como de pobre precisión (Sartory et al. 2008).

Las modificaciones metodológicas aplicadas muestran un mejor comportamiento a niveles bajos de inóculo. Se 
debe notar que ciertos protocolos de validación no recomiendan el uso de muestras con un número tan bajo de bacterias para evitar basar las interpretaciones en criterios de ausencia o presencia. (Barrell et al. 2002). Lo más importante en este caso es acercar el nivel de las muestras a los límites regulatorios vigentes, tanto a nivel nacional (Decreto № 25018-MEIC) como internacional (Abbaszadegan 2006). Los resultados en estos puntos son satisfactorios y concuerdan con resultados reportados con anterioridad por otros autores, tanto aquellas que involucran el uso de tubos múltiples de fermentación, como el empleo de técnicas de filtración de membrana. Muchos de estos estudios reportan niveles de recuperación similares o superiores a los métodos de referencia, incluyendo el Número Más Probable (Garcia-Armisen et al. 2007)

El uso de muestras naturales no evidencia una buena comparabilidad entre ambos métodos tal y como se evidenció en el Cuadro 2. Las diferentes condiciones presentes en una muestra natural producen variaciones apreciables en el rendimiento del nuevo método, siendo este más limitado para detectar los coliformes totales y fecales. Anteriormente se señaló la baja selectividad del Caldo Lactosado en fase presuntiva como una de las ventajas en la mayor recuperación de coliformes en las muestras de referencia; este aspecto, sin embargo, pareciera ser causante de que en muestras con una población más diversa de bacterias aumente la frecuencia de falsos negativos por inhibición del crecimiento de coliformes ante el incremento de otros microorganismos. Previamente se ha mencionado el problema de los medios de cultivo basados en lactosa (Public Health Laboratory Service 1980, Pitkanen et al. 2007) o tergitol (Niemela et al. 2003) para el análisis de coliformes, debido a su baja selectividad. No se tiene conocimiento claro sobres la capacidad de crecimiento de la flora microbiana normal del agua en Caldo Lactosado. El hecho de ser Costa Rica un país tropical aumenta las posibilidades de que las muestras de agua se presenten con una población bacteriana abundante (Mercado \& Hazen 1987, Isaac et al. 1998, Feldsine et al. 2002, Chao et al. 2003, Anderson et al. 2005). Otra situación es la posibilidad de falsos negativos en las muestras. Por ejemplo, se ha confirmado que Proteus vulgaris es causa de falsos negativos en muestras de agua al limitar la producción de gas de E. coli en fase presuntiva (Pouch 2001). Debe tomarse en cuenta la posibilidad de encontrar cepas de coliformes con comportamientos enzimáticos y bioquímicos inusuales, que respondan de forma muy distinta frente al medio de cultivo y las condiciones de incubación. Se conoce también que las cepas bacterianas de referencia pueden arrojar resultados atípicos en relación con los tipos salvajes (APHA 2005). Por estas mismas razones Nie- mela et al. (2003) recomiendan la confirmación de todos los resultados positivos obtenidos.

La variabilidad puede sustentarse también en la distribución aleatoria o al azar de la población bacteriana en cada una de las muestras, tomando en cuenta que esta puede verse influenciada por la composición física de la muestra, la presencia de otras bacterias (repulsión) y una mezcla inadecuada (Winward et al. 2008). Esto es una diferencia especial de tomar en cuenta en una validación microbiológica, a diferencia de una de carácter químico. Los microorganismos no son iones; forman suspensiones más que soluciones y estas suspensiones tienen un alto grado de heterogeneidad implícito que se exacerba por interacciones entre los microorganismos y partículas presentes (Sartory 2005).

Tomando en cuenta los otros factores que afectan la variabilidad del ensayo por tubos múltiples de fermentación, el sistema volumétrico debe mencionarse como importante. Con anterioridad se ha mencionado que una de las mayores fuentes de variación en el método de NMP es una deficiente preparación de alícuotas (Pouch 2001). Los resultados obtenidos en este trabajo demuestran que los volumétricos analizados poseen una buena precisión de sus resultados con base en el criterio $R$ para cada ensayo.

La validación metodológica es un proceso complejo, donde muchas variables técnicas deben incluirse. Como se aprecia en este manuscrito, el análisis de coliformes en aguas es una herramienta útil pero requiere un nivel alto de competencia técnica en su realización. El mejoramiento continuo de los métodos de ensayo por medio de la validación representa un medio efectivo para guiar las pautas de análisis en un laboratorio. Además, con experiencias como la demostrada en este artículo, los esquemas de validación se pueden mejorar y complementar para ser adaptados a las necesidades actuales de cada técnica y laboratorio (Niemela et al. 2003).

\section{REFERENCIAS}

Abbaszadegan, M. 2006.Guidelines for drinking-water quality, World Health Organization. Ginebra, Suiza.

Anderson K., J. Whitlock \& V. Harwood. 2005. Persistence and differential survival of fecal indicator bacteria in subtropical waters and sediments. Applied and Environmental Microbiology 71: 3041-3048.

American Public Health Association. 2005. Standard methods for the examination of water and wastewaters. Washington DC, EEUU.

Barrell R, C. Benton, D.Blake, P. Boyd \& S. Cole. 2002. The microbiology of drinking water-Part 3-Practices and procedures for laboratories in methods for the examination of 
waters and associated materials. Environment Agency, Washington DC, EEUU.

Beauchamp C., A. Simao-Beaunoir, C. Beaulieu \& F. Chalifour. 2006. Confirmation of E. coli among other thermotolerant coliform bacteria in paper mill effluents, wood chips screening rejects and paper sludges. Water Research 40: 2452-2462.

Chao K., C. Chao \& W. Chao. 2003. Suitability of the traditional microbial indicators and their enumerating methods in the assessment of fecal pollution of subtropical freswater environments. Journal of Microbiology, Immunology and Infectology 36: 288-293.

Clark D., B. Milner, M.Stewart, R. Wolfe \& B. Olson. 1991. Comparative study of commercial 4-Methylumbelliferyl- $\beta-D$ Glucuronide preparations with the standard methods membrane filtration fecal coliform test for the detection of Escherichia coli in water samples. Applied Environmental Microbiology. 57: 1528-1534.

Eckner K. 1998. Comparison of membrane filtration and multiple tube fermentation by the colilert and enterolert methods for detection of waterborne coliform bacteria, Escherichia coli, and enterococci used in drinking and bathing water quality monitoring in Southern Sweden. Applied Environmental Microbiology 64: 3079-3083.

Feldsine P., C. Abeyta \& W. Andrews. 2002. AOAC international methods committee guidelines for validation of qualitative and quantitative food microbiological official methods of analysis. Microbiology Guidelines. AOAC International. Maryland, EEUU.

Garcia-Armisen T., J.Prats \& P. Servais. 2007. Comparison of culturable fecal colforms and Escherichia coli enumeration in freshwaters. Canadian Journal of Microbiology 53: 798-801.

International Organization for Standardisation. ISO 17994. 2004 "Criterios para el establecimiento de equivalencia entre métodos microbiológicos". Ginebra, Suiza.

International Organization for Standardisation. ISO/TR 13843. 1996. "Guía para la validación de métodos microbiológicos en aguas". Ginebra, Suiza

International Organization for Standardisation.ISO 16140. 2005. "Protocolo para la validación de Métodos Alternativos". Ginebra, Suiza.

International Organization for Standardisation. INTE-ISO/IEC17025. 2005 "Requerimientos para la competencia de los laboratorios de ensayo y calibración." Ginebra, Suiza.

Isaac A. \& C. Lezama. 1998. Sanitary quality of water for human consumption in a rural community of Mexico. Acta Científica Venezolana 49: 187-192.
McDaniels A., R. Bordner, J. Menkedick \& C. Weber. 1987. Comparison of the hydrophobic-grid membrane filter procedure and standard methods for coliform analysis of water. Applied and Environmental Microbiology 53: 1003-1009.

Mercado J. \& T. Hazen T. 1987. Comparison of four membrane filter methods for fecal coliform enumeration in tropical waters. Applied Environmental Microbiology 53: 2922-2928.

Ministerio de Economía, Industria y Comercio de Costa Rica (MEIC). Decreto No 25018- MEIC, publicado en el Diario Oficial La Gaceta No 59 del 25 de marzo de 1996.

Moldenhauer J. 2005. Comparability Protocols. Rapid Microbiology Newsletter 3:3-7.

Niemela S., J. Lee \& C. Fricker. 2003. A comparison of the International Standards Organisation reference method for the detection of coliforms and Escherichia coli in water with a defined substrate procedure. Journal Applied Microbiology 95: 1285-1292.

Pitkanen T., P. Paakkari, I. Miettinen, H. Heinonen-Tanski, L. Paulin \& M. Hanninen. 2007. Comparison of media for enumeration of coliform bacteria and Escherichia coli in nondisinfected water. Journal Microbiological Methods 68: 522-529.

Pouch F. 2001. Compendium of Methods for the Microbiological Examination of Foods. American Public Health Association, Washington DC, EEUU.

Public Health Laboratory Service and the Standing Committee of Analysts. 1980. A comparison between minerals-modified glutamate medium and lauryl trypstose lactose broth for the enumeration of Escherichia coli and coliform organisms in water by the multiple tube method. Journal of Hygiene 85: 35-49.

Sartory, D. 2005. Validation, verification and comparison: Adopting new methods in water microbiology. Water SA 31: 393-396.

Sartory D., H. Gu \& C. Chen. 2008. Comparison of a novel MPN method against the yeast extract agar (YEA) pour plate method for the enumeration of heterotrophic bacteria from drinking water. Water Research 42: 3489-3497.

Weitzel M. 2007. How to meet ISO 17025. Requirements for Method Verification. AOAC International, Maryland, EEUU.

Winward G., L. Avery, T. Stephenson \& B.Jefferson. 2008. Chlorine disinfection of grey water for reuse: Effects of organics and particles. Water Research 42: 483-491. 
